\title{
The Deletion Polymorphism in Exon 8 of Uncoupling Protein 2 is Associated with Severe Obesity in a Saudi Arabian Case-Control Study
}

\author{
Yahia A Kaabi* \\ Department of Medical Laboratory Technology, Jazan University, Saudi Arabia
}

Received: January 30, 2018; Published: February 08, 2018

*Corresponding author: Yahia A Kaabi, Department of Medical Laboratory Technology, Jazan University, Saudi Arabia, Tel: 966 549918001; Email: ykaabi@jazanu.edu.sa

Abstract

Objectives: To establish whether 45-bp insertion (I)/deletion (D) polymorphisms in Uncoupling protein 2 (UCP2) are associated with moderate and/or severe obesity in a Saudi Arabian population.

Methods: The study enrolled 151 (male and female) subjects originating from the eastern province of Saudi Arabia, and assigned each to either a 'non-obese', 'moderately obese,' or 'severely obese' group. Genomic DNA was extracted from all subjects, and screened for UCP2 insertion/deletion polymorphisms using a standard PCR protocol.

Results: The overall frequencies of the UCP2 45-bp insertion/deletion genotypes D/D, I/D, and I/I within the analyzed population were $58.3 \%, 36.4 \%$, and 5.3\% respectively. The D/D genotype was highly prevalent within the severely obese group (82.9 \%) compared to the non-obese (46.2\%) and moderately obese (53.3\%) groups. Using a dominance model, the conducted logistic regression analysis showed no significant association between the insertion allele and moderate obesity ( $\mathrm{OR}=0.75,95 \% \mathrm{CI}$ : $0.35-1.59, \mathrm{P}=0.585)$; however, in contrast, a strong association was found between the deletion allele and severe obesity $(\mathrm{OR}=0.18,95 \% \mathrm{CI}: 0.07-0.44, \mathrm{P}=0.0004)$.

Conclusion: The present study reported the frequency of the UCP2 45-bp insertion/deletion polymorphisms in a population originating from eastern Saudi Arabia, and furthermore, identified a strong association between the D/D genotype and severe obesity in this population.

Keywords: Uncoupling protein 2 insertion/deletion polymorphism; Obesity

Abbreviations: UCP2: Uncoupling Protein 2; UTR: Untranslated Region; BMI: Body Mass Index; KFHU: King Fahad Hospital of the University; PCR: Polymerase Chain Response; SD: Standard Deviation; ORs: Odds Ratios

\section{Introduction}

Obesity is a serious and increasing prevalent medical condition in both Western and some developing countries. It is associated with a high mortality rate, caused by complications including cardiovascular and inflammatory diseases, insulin resistance, and cancer [1]. For many Gulf countries, including Saudi Arabia, obesity is becoming a major health concern for patients of both sexes [2]. In fact, this condition is estimated to affect $24.1 \%$ of men and $33.5 \%$ of women in Saudi Arabia [3]. While risk factors such as a high caloric intake and a sedentary lifestyle are known to contribute to obesity pathogenesis, it is likely that predisposing genetic factors also contribute to the development of this condition. The mitochondrial proton carrier Uncoupling protein 2 (UCP2) is a member of the mitochondrial anion carrier protein subfamily, (which also includes UCP1, 3, 4, and 5). Uncoupling proteins function to increase proton influx through the inner mitochondrial membrane without ATP synthesis, and thereby enable efficient caloric consumption and heat generation [4].

They also inhibit the production of cellular free radicals and reactive oxygen species [5]. UCP2 is the most ubiquitously expressed of these proteins, and also exerts the greatest impact on mitochondrial function; thus, it has been the most extensively studied to date. The $8 \mathrm{~kb}$ UCP2 gene sequence is located on the $\mathrm{q}$ arm of chromosome 11, and comprises eight exons, and a GC rich promoter region [6]. Three polymorphisms have been previously identified in UCP2, including i) a -866 G/A polymorphism in the promoter region [7], ii) an Ala55Val polymorphism in exon 4, and iii) a 45-bp insertion/deletion polymorphism in the 3 ' untranslated region (3' UTR) of exon 8 [8]. Identified 45 bp insertion/deletion 
polymorphisms have not been shown to impact UCP2 mRNA expression; however, they do affect UCP2 mRNA stability [8,9], and are therefore likely to also impact UCP2 function, disrupting the normal rates of metabolism and fat deposition. Initially, the insertion allele was found to be associated with an increased metabolic rate and low body mass index (BMI) in a population of Pima Indians; however, several association studies have since been conducted to evaluate whether this insertion/deletion polymorphism is associated with obesity, and/or related disorders such as type II diabetes mellitus.

For example, two meta-analysis studies published in 2014 assessed the pooled effect of the UCP2 insertion/deletion polymorphism on the rate of obesity. The first of these found no significant association between the presence of the polymorphism and obesity in either European or Asian subjects [10], while conversely, the second revealed a significant association between the insertion allele and an increased body mass index (BMI) in analyzed Asian, but not European subjects [11]. It is likely that geographical differences continue to influence the pattern of distribution of UCP2 insertion/deletion polymorphisms, as well as their relatedness to obesity. This is supported by the fact that a recent study of an Iranian population (adjacent to Saudi Arabia), found a significant association between the homozygous deletion (D/D) genotype and metabolic syndrome [12]. Thus, the current study investigated whether the UCP2 45-bp insertion/deletion polymorphisms are associated with moderate and/or severe obesity in a Saudi Arabian population.

\section{Methods}

\section{Ethics}

An informed consent was provided by all subjects for their participation in the study, whose design was approved by the institutional ethics committee (IRB-2015-03-167).

\section{Participants}

The present case-control study recruited 151 volunteer (male and female, aged 35-60 years) subjects that originated from the eastern province of Saudi Arabia, and attended the out-patient blood collection service provided by the King Fahad Hospital of the University (KFHU), at the University of Dammam, between February-July 2016. The subjects were divided into three groups, comprising
a) 'Non-obese' (BMI $\left.<30 \mathrm{~kg} / \mathrm{m}^{2}, \mathrm{n}=65\right)$,
b) 'Moderately obese' (BMI 30-35 kg/m², n=45), and
c) 'Severely obese' (BMI $\left.\geq 35 \mathrm{~kg} / \mathrm{m}^{2}, \mathrm{n}=41\right)$.

\section{Baseline Measurements}

Sociodemographic and health data for each subject (including gender, age, exhibition of type II diabetes mellitus and/or hypertension, and cigarette use), were provided via a structured questionnaire. Anthropometric data (including subject weight, height, and waist circumference) were measured using a metric and a vertical weight scale, with bare feet and only light clothing.
Each subjects BMI value was indirectly calculated according to the formula below:

$$
\text { BMI = Subject's weight }(\mathrm{kg}) /(\text { Subject's height }(\mathrm{m}))^{2}
$$

\section{Blood Collection and DNA Extraction}

Whole blood samples ( $5 \mathrm{ml}$ ) were collected in EDTA vacationer tubes via aseptic venipuncture. Genomic DNA was then extracted from the leukocytes contained in $300 \mu \mathrm{L}$ of each of these samples using an illustra blood genomic Prep Mini Spin Kit (GE Healthcare Life Sciences Ltd, UK) according to the manufacturer's instructions.

\section{Genotyping Analysis}

The presence/absence of the UCP2 45-bp insertion/deletion polymorphism was assessed via polymerase chain response (PCR) using a $2 \times$ master mix (Promega; Wisconsin, USA), $1 \mu \mathrm{L}$ of extracted template DNA, and $1 \mu \mathrm{L}(0.1 \mu \mathrm{g})$ each of forward (5' CAG TGA GGG AAG TGG GAG G 3') and reverse (5' GGG GCA GGA CGA AGA TTC $\left.3^{\prime}\right)$ primer. DNA amplification was achieved using a thermal profile comprising (initial denaturation) $4 \mathrm{~min}$ at $95^{\circ} \mathrm{C}$, followed by 35 cycles of (denaturation) $30 \mathrm{~s}$ at $93^{\circ} \mathrm{C}$, (primer binding) $30 \mathrm{~s}$ at $58^{\circ} \mathrm{C}$, and (elongation) $30 \mathrm{~s}$ at $72^{\circ} \mathrm{C}$, and a final (extension) step of $10 \mathrm{~min}$ at $72^{\circ} \mathrm{C}$. The resultant products $(10 \mu \mathrm{L})$ were then separated on a $1 \%$ agarose gel, and visualized using Ethidium bromide (EtBr) $(0.5$ $\mu \mathrm{L} /$ gel). Deletion (D) and insertion (I) alleles were identified to be present by the appearance of $412 \mathrm{bp}$ and $457 \mathrm{bp}$ bands, respectively (Figure 1).

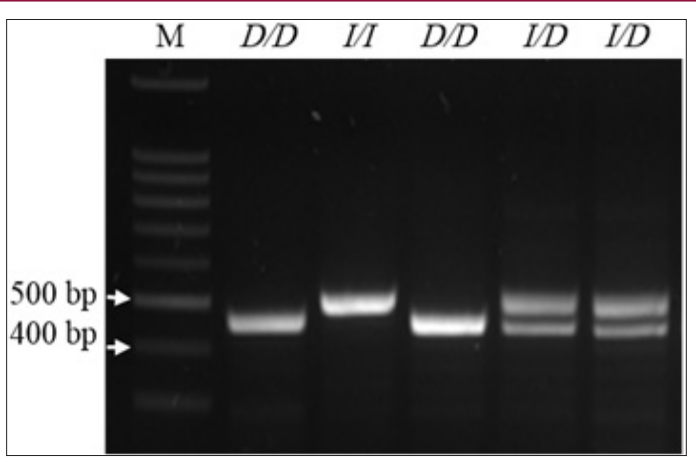

Figure 1: The presence/absence of the UCP2 45-bp insertion/deletion polymorphism was confirmed by separating PCR products on a $1 \%$ agarose gel. Lane 1, DNA ladder (M); Lane 2 and 4, the homozygous deletion (D/D) genotype was detected as a single 412 bp PCR fragment; Lane 3, the homozygous insertion genotype (I/I) was detected as a single $457 \mathrm{bp}$ PCR fragment; Lane 5 and 6, the heterozygous insertion/deletion (I/D) genotype was detected as two coincident 457 and $412 \mathrm{bp}$ fragments.

\section{Statistical Analysis}

All statistical analyses were performed using Graph Pad Prism 7 software (California, USA). Data from all study groups were presented as either the mean \pm standard deviation (SD), or as percentage values. Continuous variables were assessed using an analysis of variance (ANOVA) test, whereas categorical variables were compared using a either a Chi-square or Fisher's exact test. Potential associations between the UCP2 insertion/deletion polymorphisms and obesity were evaluated by calculating the odds 
ratios (ORs), 95\% confidence intervals, and two-sided Chi-square test and/or Yates' correction P values to conduct logistic regression analyses. A logistic binary regression was used to adjust all ORs to confound parameters such as subject age and sex. A P value less than 0.05 was considered to indicate statistical significance.

\section{Results}

The demographic characteristics of the study population are summarized in Table 1 . The mean age of the study population was found to be $44.78 \pm 10.31$ years, and no statistical difference in age was observed between the three study groups $(\mathrm{P}=0.1023)$. The overall male/female ratio, which was $72 / 79$ across the three study groups, was reduced in both the moderately and the severely obese subject groups compared to the non-obese group; however, these differences were not statistically significant $(P=0$. 1663). The mean ( \pm standard deviation) BMI values for the non-, moderately, and severely obese subject groups were calculated to be $26.18 \pm$ $1.92,32 \pm 1.83$, and $39.59 \pm 3.48$ respectively, and shown to be significantly different via the conducted ANOVA test $(\mathrm{P}=<0.0001)$. Type II diabetes mellitus and hypertension were more prevalent in the moderately (affecting $40 \%$ and $28.9 \%$ of subjects, respectively) and the severely obese (affecting 51.1\% and 53.65\% of subjects, respectively) groups than in the non-obese group (affecting $29.2 \%$ and $18.5 \%$ of subjects, respectively). In contrast, the frequency of cigarette use was increased in the non-obese group $(27.7 \%)$ compared to the moderately (13.3\%) and severely obese $(12.2 \%)$ groups.

Table 1: Baseline characteristic of the study population, $(n=151)$.

\begin{tabular}{|c|c|c|c|c|}
\hline & $\begin{array}{l}\text { Non-obese }\left(\mathrm{BMI}<30 \mathrm{~kg} / \mathrm{m}^{2}\right) \\
(\mathrm{n}=65)\end{array}$ & $\begin{array}{c}\text { Moderately obese (BMI } \geq 30 \\
\left.\mathrm{~kg} / \mathrm{m}^{2}\right)(\mathrm{n}=45)\end{array}$ & $\begin{array}{c}\text { Severely obese (BMI >35 } \\
\left.\mathrm{kg} / \mathrm{m}^{2}\right)(\mathrm{n}=41)\end{array}$ & $\mathbf{P}^{*}$ \\
\hline Gender (male/female) & $36 / 29$ & $21 / 24$ & $15 / 26$ & 0.1663 \\
\hline Age (years) & $46.33 \pm 11.12$ & $45.49 \pm 9.14$ & $42.53 \pm 10.67$ & 0.1023 \\
\hline Height $(\mathrm{m})$ & $163.5 \pm 7.99$ & $164.4 \pm 8.05$ & $160.10 \pm 10.37$ & 0.4352 \\
\hline Weight (kg) & $70.01 \pm 8.55$ & $83.08 \pm 8.71$ & $102.1 \pm 17.61$ & $<0.0001$ \\
\hline Waist circumference $(\mathrm{cm})$ & $92.66 \pm 8.10$ & $106.9 \pm 5.369$ & $118.5 \pm 12.43$ & $<0.0001$ \\
\hline BMI $\left(\mathrm{kg} / \mathrm{m}^{2}\right)$ & $26.18 \pm 1.92$ & $32 \pm 1.83$ & $39.59 \pm 3.48$ & $<0.0001$ \\
\hline Smoking n (\%) & $18(27.69)$ & $6(13.33)$ & $5(12.19)$ & 0.0701 \\
\hline Diabetes n (\%) & $19(29.23)$ & $18(40.00)$ & $23(51.11)$ & 0.0226 \\
\hline Hypertension n (\%) & $12(18.46)$ & $13(28.88)$ & $22(53.65)$ & 0.0006 \\
\hline
\end{tabular}

The UCP2 45-bp insertion (I)/deletion (D) genotypes D/D, I/D, and I/I were detected to occur with a frequency of $58.3 \%, 36.4 \%$, and $5.3 \%$ across the total study population, respectively, while the deletion and insertion alleles were detected in $76.4 \%$ and $23.6 \%$ of study subjects, respectively. The D/D genotype was found to be more highly prevalent in the severely obese group (82.9\%) compared to the non-obese $(46.2 \%)$ and moderately obese (53.3\%) groups. Table 2: Association of the UCP2 54-bp insertion (I)/deletion (D) polymorphisms with obesity.

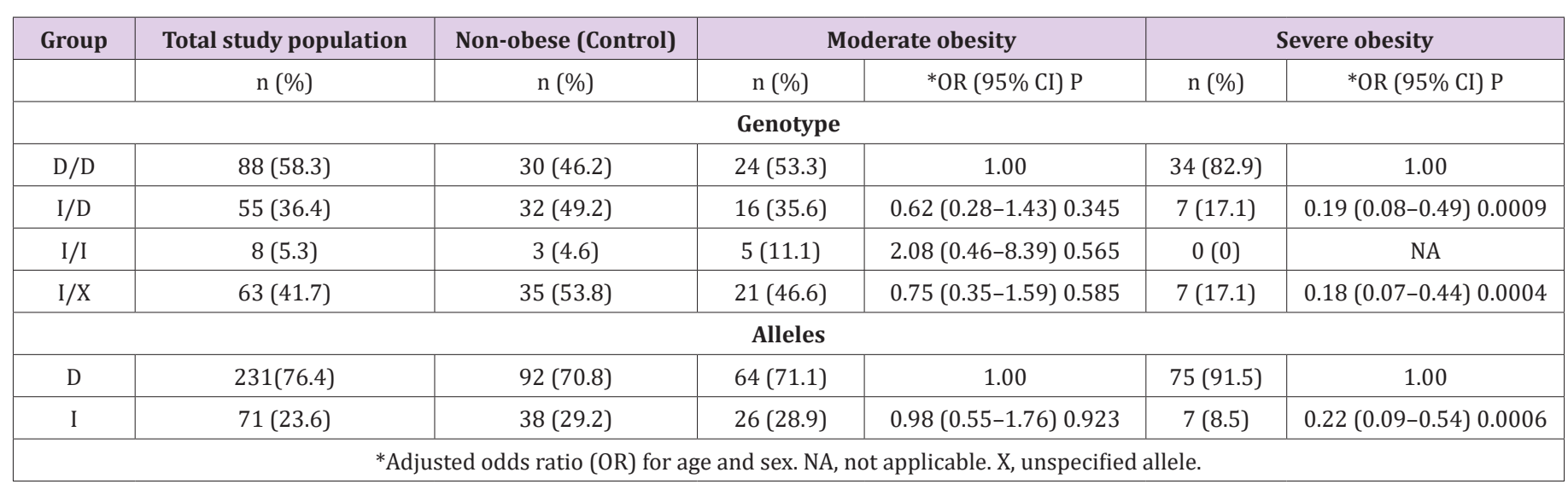

\section{Discussion}

The role of the 45-bp insertion/deletion polymorphism in exon 8 UCP2 gene in the development of obesity is to date, unclear. Thus, the current study investigated the frequency of this polymorphism
The conducted logistic regression analysis showed no significant association between the I/X genotypes (where X represents a nonspecified allele) and the development of moderate obesity (OR $=0.75,95 \% \mathrm{CI}: 0.35-1.59, \mathrm{P}=0.585$ ); however, a strong inverse association was found between these genotypes and severe obesity $(\mathrm{OR}=0.18,95 \%$ CI: $0.07-0.44, \mathrm{P}=0.0004)$ (Table 2). 
either an 'overweight' BMI $\left(25-30 \mathrm{~kg} / \mathrm{m}^{2}\right)$ or type II diabetes mellitus (data not shown), a strong association was identified between the $\mathrm{D} / \mathrm{D}$ genotype and severe obesity in this population. In fact, subjects with this genotype were found to experience a fivetime greater risk for developing severe obesity that than those who harbored at least one insertion allele. The majority of previously conducted association studies have reportedly found no direct link between the UCP2 45-bp insertion/deletion polymorphism and either obesity or various related metabolic disorders [13-18]. Notably, some previous studies showed the insertion allele to be positively associated with obesity, contradictory to the findings of the present study [19-22]. Nevertheless, various studies have produced results consistent with those presented here, suggesting that the D/D genotype may contribute to the development of obesity and its related metabolic disorders.

For instance, the deletion allele has been previously associated with lower resting metabolic and energy expenditure rates in young adults, and thus postulated to contribute to the development of obesity at a later age $[8,23]$. Similarly, subjects carrying the insertion allele in a (metabolically healthy) Greek population were previously shown to exhibit improved weight loss profiles compared to those harboring the D/D genotype [18]. Previous screening of a Tongan population with a known increasing prevalence of obesity revealed the D/D genotype to be highly prevalent (97\%) [24]. Finally, a recent study in the region adjacent to the population analyzed by the present study reported a significant association between the D/D deletion and metabolic syndrome, for which obesity is a wellknown risk factor [12]. In Saudi Arabia, obesity is a common health concern caused by excessive caloric intake and/or low physical activity levels. The reduced metabolic and energy expenditure rates incurred by the $\mathrm{D} / \mathrm{D}$ genotype likely aggravate the tendency to develop severe obesity in this population [25].

Notably, previous analysis of a western Saudi Arabian population showed the insertion/deletion polymorphism to be neither associated with obesity nor type II diabetes mellitus [26]. This discrepancy may reflect the fact that this previous study did not consider moderately and severely obese subjects separately, or alternatively, may reflect genetic differences incurred by geographical separation of the two populations. In conclusion, the present study showed the distribution of the UCP2 D/D, D/I, and I/I genotypes in the analyzed eastern Saudi Arabian population to be $58.3 \%, 36.4 \%$, and 5.3\%, respectively. Moreover, the D/D genotype was shown to be strongly associated with severe, but not moderate obesity. These finding provide valuable insights into the genetic contribution of UCP2 to obesity and related disorders in Saudi Arabia, and should be confirmed via additional research with larger and more geographically diverse cohorts.

\section{Acknowledgement}

I take this opportunity to thank Mrs Amani Al Omairy for her great support to conduct this research.

\section{References}

1. Abdelaal M, CW le Roux, NG Docherty (2017) Morbidity and mortality associated with obesity. Ann Transl Med 5(7): 161.
2. Sultan AL (2014) Obesity in gulf countries. Int J Health Sci (Qassim) 8(1): 79-83.

3. Memish ZA (2014) Obesity and associated factors-Kingdom of Saudi Arabia. Prev Chronic Dis 11: 174

4. Brand MD, TC Esteves (2005) Physiological functions of the mitochondrial uncoupling proteins UCP2 and UCP3. Cell Metab 2(2): 85-93.

5. Murphy MP (2003) Superoxide activates uncoupling proteins by generating carbon-centered radicals and initiating lipid peroxidation: Studies using a mitochondria-targeted spin trap derived from alphaphenyl-N-tert-butylnitrone. J Biol Chem 278(49): 48534-48545.

6. Pecqueur C (1999) Functional organization of the human uncoupling protein-2 gene, and juxtaposition to the uncoupling protein-3 gene. Biochem Biophys Res Commun 255(1): 40-46.

7. Enerback S (1997) Mice lacking mitochondrial uncoupling protein are cold-sensitive but not obese. Nature 387(6628): 90-94.

8. Walder K (1998) Association between uncoupling protein polymorphisms (UCP2-UCP3) and energy metabolism/obesity in Pima indians. Hum Mol Genet 7(9): 1431-1435.

9. Lindholm E (2004) Putative role of polymorphisms in UCP1-3 genes for diabetic nephropathy. J Diabetes Complications 18(2): 103-107.

10. Zhang M, M Wang, ZT Zhao (2014) Uncoupling protein 2 gene polymorphisms in association with overweight and obesity susceptibility: A meta-analysis. Meta Gene 2: 143-159.

11. Brondani LA (2014) Meta-analysis reveals the association of common variants in the uncoupling protein (UCP) 1-3 genes with body mass index variability. PLoS One 9(5): e96411.

12. Hashemi M (2014) A 45-bp insertion/deletion polymorphism of UCP2 gene is associated with metabolic syndrome. Journal of Diabetes \& Metabolic Disorders 13(1): 12.

13. Dalgaard LT (1999) An untranslated insertion variant in the uncoupling protein 2 gene is not related to body mass index and changes in body weight during a 26-year follow-up in Danish Caucasian men. Diabetologia 42(12): 1413-1416.

14. Maestrini S (2003) Lack of association between UCP2 gene polymorphisms and obesity phenotype in Italian Caucasians. Journal of Endocrinological Investigation 26(10): 985-990.

15. Ochoa MC (2007) Association between obesity and insulin resistance with UCP2-UCP3 gene variants in Spanish children and adolescents. Mol Genet Metab 92(4): 351-358.

16. Yiew SK (2010) No association between peroxisome proliferatoractivated receptor and uncoupling protein gene polymorphisms and obesity in Malaysian university students. Obes Res Clin Pract 4(4): 247342 .

17. Liu X (2012) A 45-bp insertion/deletion polymorphism in uncoupling protein 2 is not associated with obesity in a Chinese population. Biochem Genet 50(9-10): 784-796.

18. Papazoglou D (2012) Uncoupling protein-2 45-base pair insertion/ deletion polymorphism: is there an association with severe obesity and weight loss in morbidly obese subjects?. Metab Syndr Relat Disord 10(4): 307-311.

19. Cassell PG (1999) An uncoupling protein 2 gene variant is associated with a raised body mass index but not Type II diabetes. Diabetologia 42(6): 688-692.

20. Evans D (2000) Frequency of and interaction between polymorphisms in the beta3-adrenergic receptor and in uncoupling proteins 1 and 2 and obesity in Germans. Int J Obes Relat Metab Disord 24(10): 1239-1245.

21. Marti A (2004) Higher obesity risk associated with the exon-8 insertion of the UCP2 gene in a Spanish case-control study. Nutrition 20(6): 498501. 
22. Lee YH (2008) Association of the ins/del polymorphisms of uncoupling protein 2 (UCP2) with BMI in a Korean population. Biochem Biophys Res Commun 371(4): 767-771.

23. Kovacs $P$ (2005) Genetic variation in UCP2 (uncoupling protein-2) is associated with energy metabolism in Pima Indians. Diabetologia 48(11): 2292-2295.

24. Duarte NL (2003) A 45-bp insertion/deletion polymorphism of uncoupling protein 2 in relation to obesity in Tongans. Obes Res, 11(4): 512-517.

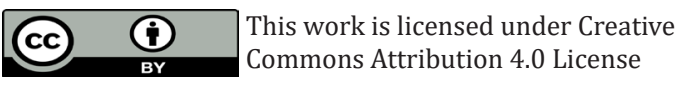

Submission Link: http://biomedres.us/submit-manuscript.php
25. De Nicola E (2015) Obesity and public health in the Kingdom of Saudi Arabia. Rev Environ Health, 30(3): 191-205.

26. Jiffri E (2012) Association of the UCP2 45-bp insertion/deletion polymorphism with diabetes type 2 and obesity in Saudi population. Egyptian Journal of Medical Human Genetics 13(3): 257-262.

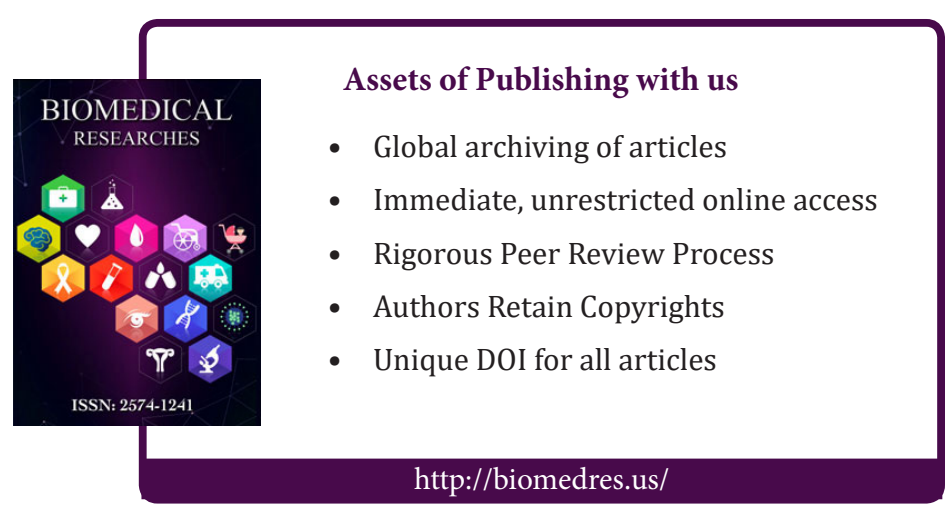

